\title{
Comparison of Coupled Mode Theory and FDTD Simulations of Coupling between Bent and Straight Optical Waveguides
}

\author{
Remco Stoffer, Kirankumar R. Hiremath and Manfred Hammer \\ $\mathrm{MESA}^{+}$Research Institute, University of Twente, The Netherlands
}

\begin{abstract}
Analysis of cylindrical integrated optical microresonators involves the coupling between a straight waveguide and a bent waveguide. Our (2D) variant of coupled mode theory is based on analytically represented mode profiles. With the bend modes expressed in Cartesian coordinates, coupled mode equations can be derived in a classical way, and solved by numerical integration. Proper manipulation of the propagation matrix leads to stable results that satisfy the reciprocity properties for symmetric coupler devices, even in parameter domains of rather compact, radiative structures and strong interaction, which before seemed unaccessible by a perturbational approach. Comparisons with FDTD calculations show convincing agreement.
\end{abstract}

\section{INTRODUCTION}

Along with the current trend towards large-scale integration in photonics [1], accurate and efficient design tools for small and densely packed components are required. Arrays of optical microring resonators (MR) [2] are among the concepts at the focus of the research. For this paper we consider single resonator elements, consisting of a circular, ring- or disk-shaped cavity that is evanescently coupled to two straight waveguides. Rigorous numerical simulations of such devices are possible, e.g. by means of Finite Difference Time Domain (FDTD) methods [3], but usually those direct calculations are very time-consuming. Alternatively, one can divide the device into separate regions, model those individually, and combine the parts into a complete resonator model. The MR can be separated into four parts: The ring itself, represented by two segments of a bent waveguide, and the two couplers involving a straight waveguide and a bent waveguide. Here we will focus on the coupler part. For a discussion of the extension of these coupler simulations towards a full ringresonator description we refer to Refs. $[4,5]$.

For the coupler region, it is again possible to do direct FDTD calculations to determine at least part of the coupler characteristics. While this works for all configurations, even those that are highly radiative, the calculation times involved are still relatively high; especially in three-dimensional simulations the computational effort becomes unacceptable. For a detailed design parameter analysis, a fast tool is needed to determine the elements of the coupler scattering matrix that enter the abstract resonator model [5]. A good candidate is the well-known Coupled Mode Theory (CMT); see e.g. [6, $7,8,4]$ for the general theory, [9] and [10] for an application to microresonators, or [11] for a preliminary version of the implementation described in this paper. For this implementation we transform the bend field to Cartesian coordinates and apply a more or less standard CMT formulation in a rectangular domain. While the theory is as valid 

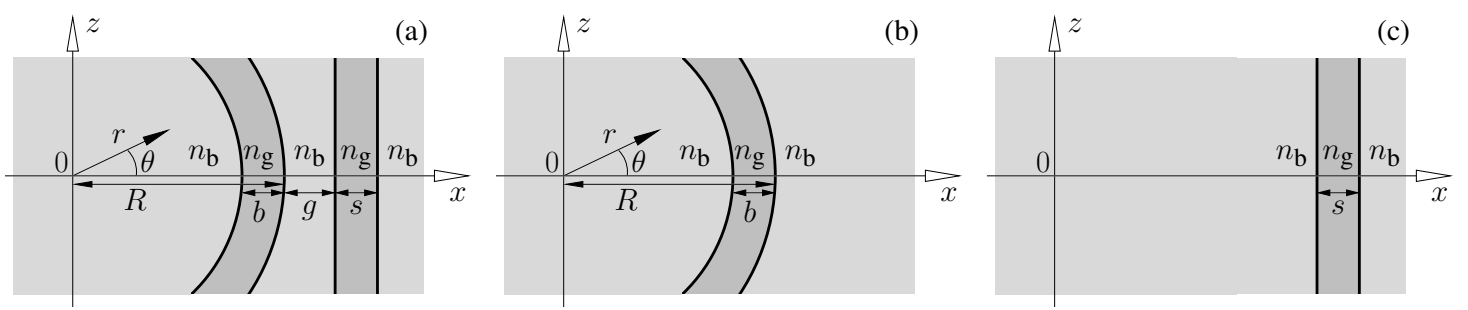

FIGURE 1. Schematic coupler configuration involving a bent waveguide and a straight waveguide (a). The straight core of width $s$ is evanescently coupled to the bent waveguide of width $b$ and radius $R$, separated by a gap distance $g$. Refractive indices $n_{\mathrm{g}}$ and $n_{\mathrm{b}}$ characterize the guiding regions and the background material. For purposes of modeling in the framework of coupled mode theory, the structure is considered to be composed of two parts, the isolated bent (b) and straight cores (c). All numerical examples are computed with default parameters $b=s=1.0 \mu \mathrm{m}, n_{\mathrm{b}}=1.45, n_{\mathrm{g}}=1.6, \mathrm{~g} \in[0.1,1.5] \mu \mathrm{m}$, $R \in[15,500] \mu \mathrm{m}$, and a vacuum wavelength $\lambda=1.55 \mu \mathrm{m}$.

in $3 \mathrm{D}$ as it is in $2 \mathrm{D}$, we will restrict to two spatial coordinates, where our own FDTD program is available for a numerical assessment of the CMT results.

Section 2 gives an outline of the present CMT formulation. In Section 2.1 relations between the solutions of the coupled mode equations and the the entries of the coupler scattering matrix are established. It turns out that the correct extraction of the coefficients related to the waves in the straight waveguide requires a projection onto the basis mode of the straight core. This is the subject of Section 2.2. Further numerical results concerning the comparison of coupling coefficients computed by FDTD and CMT and a discussion of reciprocity properties are presented in Section 3.

\section{COUPLED MODE THEORY}

A harmonic time dependence $\sim \mathrm{e}^{\mathrm{i} \omega t}$ with angular frequency $\omega=2 \pi c / \lambda$ and vacuum wavelength $\lambda$ is assumed for all fields. We use a Cartesian coordinate system $(x, y, z)$ as a reference system. In the 2D approach, the field and materials are assumed to be constant in the $y$-direction. The analysis is restricted to TE fields. We assume that all individual waveguides are monomodal, waves are forward propagating, and back-reflections are negligible (cf. the discussion of the underlying resonator model in Ref. [5]).

Consider the coupler setting as shown in Figure 1. Let $\left\{\boldsymbol{E}_{\mathrm{b}}, \boldsymbol{H}_{\mathrm{b}}\right\}$ be the electromagnetic field associated with the bent waveguide (b) with $\varepsilon_{\mathrm{b}}$ as the relative permittivity distribution. Correspondingly $\left\{\boldsymbol{E}_{\mathrm{s}}, \boldsymbol{H}_{\mathrm{s}}\right\}$ and $\varepsilon_{\mathrm{s}}$ are the field and permittivity associated with the straight waveguide (c). Note that $\varepsilon_{\mathrm{s}}$ and $\varepsilon_{\mathrm{b}}$ are the complete permittivity distributions, i.e. functions defined on the $x-z$ plane. Then we write the following ansatz for the total electric field $\boldsymbol{E}$ and magnetic field $\boldsymbol{H}$ in the coupled structure (a):

$$
\boldsymbol{E}(x, z)=a_{\mathrm{b}}(z) \boldsymbol{E}_{\mathrm{b}}(x, z)+a_{\mathrm{s}}(z) \boldsymbol{E}_{\mathrm{s}}(x, z) \quad \boldsymbol{H}(x, z)=a_{\mathrm{b}}(z) \boldsymbol{H}_{\mathrm{b}}(x, z)+a_{\mathrm{s}}(z) \boldsymbol{H}_{\mathrm{s}}(x, z) .
$$

Here $a_{\mathrm{b}}(z)$ and $a_{\mathrm{s}}(z)$ are the - so far unknown - amplitudes of the bent and straight waveguide modes, respectively.

The bend mode field is determined by solving the relevant Helmholtz equation in cylindrical coordinates (see Refs. [11, 5] for details). An eigenmode solver, based on routines for the numerical evaluation of complex order Bessel and Hankel functions is 
employed to calculate the angular propagation constants, after which the radial field is known analytically. In cylindrical coordinates $(r, \theta)$ as introduced in Figure 1, the bend mode field is given as

$$
\boldsymbol{E}_{\mathrm{b}}(r, \theta)=\boldsymbol{E}_{\mathrm{b}}^{0}(r) \mathrm{e}^{-\mathrm{i} \gamma_{\mathrm{b}} R \theta}, \quad \boldsymbol{H}_{\mathrm{b}}(r, \theta)=\boldsymbol{H}_{\mathrm{b}}^{0}(r) \mathrm{e}^{-\mathrm{i} \gamma_{\mathrm{b}} R \theta},
$$

where $\gamma_{\mathrm{b}}=\beta_{\mathrm{b}}-\mathrm{i} \alpha_{\mathrm{b}}$ is the complex (angular) propagation constant, consisting of the phase constant $\beta_{\mathrm{b}}$ and the attenuation constant $\alpha_{\mathrm{b}}$. The bend radius $R$ is here defined as the distance from the origin to the outer rim of the bend (other choices are possible [5]). $\boldsymbol{E}_{\mathrm{b}}^{0}$ and $\boldsymbol{H}_{\mathrm{b}}^{0}$ are the electric and magnetic parts of the (fundamental) bend mode profile. Written out in components, the 2D TE bend mode field reads:

$$
\begin{aligned}
& E_{\mathrm{b}, r}(r, \theta)=0, \\
& E_{\mathrm{b}, y}(r, \theta)=E_{\mathrm{b}, y}^{0}(r) \mathrm{e}^{-i \gamma_{\mathrm{b}} R \theta}, \\
& H_{\mathrm{b}, r}(r, \theta)=\frac{-i}{\mu_{0} \omega r} \partial_{\theta} E_{\mathrm{b}, y}(r, \theta), \\
& E_{\mathrm{b}, \theta}(r, \theta)=0 \text {, } \\
& H_{\mathrm{b}, y}(r, \theta)=0 \text {, } \\
& H_{\mathrm{b}, \theta}(r, \theta)=\frac{i}{\mu_{0} \omega} \partial_{r} E_{\mathrm{b}, y}(r, \theta) \text {. }
\end{aligned}
$$

The profile is determined completely by the single electric component $E_{\mathrm{b}, y}$. The field can be transformed from cylindrical to Cartesian coordinates:

$$
\begin{array}{ll}
E_{\mathrm{b}, x}(x, z)=0, & H_{\mathrm{b}, x}(x, z)=\cos \theta H_{\mathrm{b}, r}(r, \theta)-\sin \theta H_{\mathrm{b}, \theta}(r, \theta), \\
E_{\mathrm{b}, y}(x, z)=E_{\mathrm{b}, y}(r, \theta), & H_{\mathrm{b}, y}(x, z)=0 \\
E_{\mathrm{b}, z}(x, z)=0, & H_{\mathrm{b}, z}(x, z)=\sin \theta H_{\mathrm{b}, r}(r, \theta)+\cos \theta H_{\mathrm{b}, \theta}(r, \theta) .
\end{array}
$$

with $r(x, z)=\sqrt{\left(x^{2}+z^{2}\right)}$ and $\theta(x, z)=\arctan z / x$. Henceforth without loss of generality, the above field components are treated as a function of the Cartesian coordinates $(x, z)$.

The electromagnetic field in the straight waveguide is given by

$$
\boldsymbol{E}_{\mathrm{s}}(x, z)=\boldsymbol{E}_{\mathrm{s}}^{0}(x) \mathrm{e}^{-\mathrm{i} \beta_{\mathrm{s}} z}, \quad \boldsymbol{H}_{\mathrm{s}}(x, z)=\boldsymbol{H}_{\mathrm{s}}^{0}(x) \mathrm{e}^{-\mathrm{i} \beta_{\mathrm{s}} z},
$$

where $\beta_{\mathrm{s}}$ is the propagation constant and the superscript zero denotes the (fundamental) mode profile. In 2D and for TE polarization, the components are:

$$
\begin{array}{ll}
E_{\mathrm{s}, x}(x, z)=0, & H_{\mathrm{s}, x}(x, z)=\frac{-i}{\mu_{0} \omega} \partial_{z} E_{\mathrm{s}, y}(x, z), \\
E_{\mathrm{s}, y}(x, z)=E_{\mathrm{s}, y}^{0}(x) \mathrm{e}^{-i \beta_{\mathrm{s}} z}, & H_{\mathrm{s}, y}(x, z)=0, \\
E_{\mathrm{s}, z}(x, z)=0, & H_{\mathrm{s}, z}(x, z)=\frac{i}{\mu_{0} \omega} \partial_{x} E_{\mathrm{s}, y}(x, z) .
\end{array}
$$

For any two electromagnetic fields $\left\{\boldsymbol{E}_{p}, \boldsymbol{H}_{p}\right\}$ and $\left\{\boldsymbol{E}_{q}, \boldsymbol{H}_{q}\right\}$, with their associated permittivity distributions $\varepsilon_{p}$ and $\varepsilon_{q}$, one can use Maxwell's equations to derive the following identity known as Lorentz Reciprocity Theorem:

$$
\int \nabla \cdot\left(\boldsymbol{E}_{p} \times \boldsymbol{H}_{q}^{*}+\boldsymbol{E}_{q}^{*} \times \boldsymbol{H}_{p}\right) \mathrm{d} x=-\mathrm{i} \omega \varepsilon_{0} \int \boldsymbol{E}_{q}^{*} \cdot\left(\varepsilon_{p}-\varepsilon_{q}\right) \boldsymbol{E}_{p} \mathrm{~d} x
$$




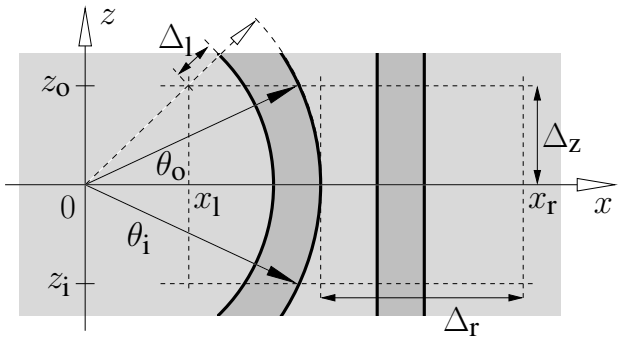

FIGURE 2. Definition of the computational window for the CMT implementation. The CMT equations are integrated along the $z$-axis between $z_{\mathrm{i}}$ and $z_{\mathrm{o}}$, over a lateral interval extending from $x_{1}$ to $x_{\mathrm{r}}$. Input- and output angles $\theta_{\mathrm{i}}$ and $\theta_{\mathrm{o}}$ are associated with the input and output planes at $z_{\mathrm{i}}$ and $z_{\mathrm{o}}$. The quantities $\Delta_{\mathrm{z}}, \Delta_{\mathrm{l}}, \Delta_{\mathrm{r}}$ serve to specify the computational window (see Section 3 ).

Let $\varepsilon$ be the permittivity distribution of the composite structure. Using Eq. (7) once with $\{\boldsymbol{E}, \boldsymbol{H}, \varepsilon\}$ and $\left\{\boldsymbol{E}_{\mathrm{b}}, \boldsymbol{H}_{\mathrm{b}}, \varepsilon_{\mathrm{b}}\right\}$, then with $\{\boldsymbol{E}, \boldsymbol{H}, \varepsilon\}$ and $\left\{\boldsymbol{E}_{\mathrm{s}}, \boldsymbol{H}_{\mathrm{s}}, \varepsilon_{\mathrm{s}}\right\}$, finally four times with $\left\{\boldsymbol{E}_{p}, \boldsymbol{H}_{p}, \boldsymbol{\varepsilon}_{p}\right\}$ and $\left\{\boldsymbol{E}_{q}, \boldsymbol{H}_{q}, \boldsymbol{\varepsilon}_{q}\right\}, p, q=\mathrm{b}, \mathrm{s}$ (the last step removes the $z$-derivatives of the overlap integrals), we arrive at the coupled mode equations:

$$
\left(\begin{array}{cc}
\sigma_{\mathrm{bb}} & \sigma_{\mathrm{bs}} \\
\sigma_{\mathrm{sb}} & \sigma_{\mathrm{ss}}
\end{array}\right) \frac{\mathrm{d}}{\mathrm{d} z}\left(\begin{array}{c}
a_{\mathrm{b}} \\
a_{\mathrm{s}}
\end{array}\right)=\left(\begin{array}{cc}
c_{\mathrm{bb}} & c_{\mathrm{bs}} \\
c_{\mathrm{sb}} & c_{\mathrm{ss}}
\end{array}\right)\left(\begin{array}{c}
a_{\mathrm{b}} \\
a_{\mathrm{s}}
\end{array}\right)
$$

with power coupling coefficients $\sigma_{p q}$ that are given by the overlap integrals

$$
\sigma_{p q}=\left\langle\boldsymbol{E}_{p}, \boldsymbol{H}_{p} \mid \boldsymbol{E}_{q}, \boldsymbol{H}_{q}\right\rangle=\frac{1}{4} \int \mathbf{e}_{z} \cdot\left(\boldsymbol{E}_{q} \times \boldsymbol{H}_{p}^{*}+\boldsymbol{E}_{p}^{*} \times \boldsymbol{H}_{q}\right) \mathrm{d} x
$$

(here $\mathbf{e}_{z}$ is a unit vector along the $z$-axis), and with coupling coefficients

$$
c_{p q}=-\mathrm{i} \frac{\omega \varepsilon_{0}}{4} \int \boldsymbol{E}_{p}^{*} \cdot\left(\varepsilon-\varepsilon_{q}\right) \boldsymbol{E}_{q} \mathrm{~d} x, \quad p, q=\mathrm{b}, \mathrm{s} .
$$

Note that apart from $\sigma_{\mathrm{ss}}$ and $c_{\mathrm{ss}}$ all coefficients are $z$-dependent.

Solving the above system of ordinary differential equations using a numerical quadrature rule for the integrals along $x$ and a fourth order Runge Kutta scheme [12] for the integration along $z$ (see Figure 2), we obtain a linear relation

$$
\left(\begin{array}{c}
a_{\mathrm{b}}\left(z_{\mathrm{o}}\right) \\
a_{\mathrm{s}}\left(z_{\mathrm{o}}\right)
\end{array}\right)=\left(\begin{array}{ll}
T_{\mathrm{bb}} & T_{\mathrm{bs}} \\
T_{\mathrm{sb}} & T_{\mathrm{ss}}
\end{array}\right)\left(\begin{array}{c}
a_{\mathrm{b}}\left(z_{\mathrm{i}}\right) \\
a_{\mathrm{s}}\left(z_{\mathrm{i}}\right)
\end{array}\right)
$$

between the coupled mode amplitudes $a_{p}\left(z_{\mathrm{i}}\right)$ on an input plane $z_{\mathrm{i}}$ and the amplitudes $a_{p}\left(z_{\mathrm{o}}\right)$ on an output plane $z_{\mathrm{o}}$. Here $\mathrm{T}=\left(T_{p q}\right)$ is the propagation matrix of the coupler. Due to the linearity of the problem, the procedure can be formulated for $T$ directly.

\subsection{Coupler scattering matrix}

The theory outlined above yields the amplitudes of the complete ansatz fields, including the exponential dependences on the propagation angle or the distance, respectively. In order to properly relate the amplitudes of the modal profiles at the start and the end of the computational window, the propagation matrix elements have to be adjusted to compensate for phase velocities and decay constants.

Consider the setting as shown in Figure 2. The coupler is defined in the region $\left[x_{l}, x_{r}\right] \times$ $\left[z_{\mathrm{i}}, z_{\mathrm{o}}\right]$. Outside this region, one assumes the interaction between the two waveguides to 
be negligible. Two input and two output ports are identified, given by the external bend or straight waveguide segments. Within these ports, the incoming or outgoing (electrical) fields are

$$
\begin{array}{ll}
A_{\mathrm{b}}^{\mathrm{o}} \boldsymbol{E}_{\mathrm{b}}^{0}(r) \mathrm{e}^{-\mathrm{i} \gamma_{\mathrm{b}} R\left(\theta-\theta_{\mathrm{o}}\right)}, \theta \geq \theta_{\mathrm{o}}, & A_{\mathrm{s}}^{\mathrm{o}} \boldsymbol{E}_{\mathrm{s}}^{0}(x) \mathrm{e}^{-\mathrm{i} \beta_{\mathrm{s}}\left(z-z_{\mathrm{o}}\right)}, z \geq z_{\mathrm{o}}, \\
A_{\mathrm{b}}^{\mathrm{i}} \boldsymbol{E}_{\mathrm{b}}^{0}(r) \mathrm{e}^{-\mathrm{i} \gamma_{\mathrm{b}} R\left(\theta-\theta_{\mathrm{i}}\right)}, \theta \leq \theta_{\mathrm{i}}, & A_{\mathrm{s}}^{\mathrm{i}} \boldsymbol{E}_{\mathrm{s}}^{0}(x) \mathrm{e}^{-\mathrm{i} \beta_{\mathrm{s}}\left(z-z_{\mathrm{i}}\right)}, z \leq z_{\mathrm{i}},
\end{array}
$$

written as appropriate in cylindrical or Cartesian coordinates, with constant external amplitudes $A_{p}^{\mathrm{i}}, A_{p}^{\mathrm{o}}$. Within the coupler region the field is given by the coupled mode ansatz (1).

The abstract model of Ref. [5] requires a scattering matrix that relates the external output amplitudes $A_{\mathrm{b}}^{\mathrm{o}}, A_{\mathrm{s}}^{\mathrm{o}}$ of the mode profiles at $z=z_{\mathrm{o}}$ to the external input amplitudes $A_{\mathrm{b}}^{\mathrm{i}}, A_{\mathrm{s}}^{\mathrm{i}}$ at $z=z_{\mathrm{i}}$. By matching the coupled and uncoupled fields at the interface planes of the computational window, one obtains

$$
\left(\begin{array}{l}
A_{\mathrm{b}}^{\mathrm{o}} \\
A_{\mathrm{s}}^{\mathrm{O}}
\end{array}\right)=\left(\begin{array}{ll}
S_{\mathrm{bb}}^{0} & S_{\mathrm{bs}}^{0} \\
S_{\mathrm{sb}}^{0} & S_{\mathrm{ss}}^{0}
\end{array}\right)\left(\begin{array}{c}
A_{\mathrm{b}}^{\mathrm{i}} \\
A_{\mathrm{s}}^{\mathrm{i}}
\end{array}\right) \quad \text { with } \quad \mathrm{S}^{0}=\mathrm{O}_{\mathrm{o}}^{0} \mathrm{~T}\left(\mathrm{O}_{\mathrm{i}}^{0}\right)^{-1}
$$

and

$$
\mathrm{O}_{\mathrm{i}}^{0}=\left(\begin{array}{cc}
\mathrm{e}^{-\mathrm{i} \gamma_{\mathrm{b}} R \theta_{\mathrm{i}}} & 0 \\
0 & \mathrm{e}^{-\mathrm{i} \beta_{\mathrm{s}} z_{\mathrm{i}}}
\end{array}\right), \quad \mathrm{O}_{\mathrm{o}}^{0}=\left(\begin{array}{cc}
\mathrm{e}^{-\mathrm{i} \gamma_{\mathrm{b}} R \theta_{\mathrm{o}}} & 0 \\
0 & \mathrm{e}^{-\mathrm{i} \beta_{\mathrm{s}} z_{\mathrm{o}}}
\end{array}\right)
$$

for the relation between $T$ and the (preliminary version of the) scattering matrix $S^{0}=$ $\left(S_{p q}^{0}\right)$ of the coupler.

As introduced so far, the elements of $\mathrm{T}$ and $\mathrm{S}^{0}$ are static quantities, defined for a fixed computational window according to Figure 2. In order to assess the interaction between the two waveguides, we consider the evolution $\mathrm{T}(z), \mathrm{S}^{0}(z)$ as obtained by applying Eqs. (11), (14) to a series of computational windows with fixed lower boundary $z_{\mathrm{i}}$ and varying upper boundary at $z \in\left[z_{\mathrm{i}}, z_{\mathrm{o}}\right]$. Then $\left|S_{\mathrm{ss}}^{0}(z)\right|^{2}$ and $\left|S_{\mathrm{bs}}^{0}(z)\right|^{2}$ can be interpreted as the local relative power fractions that are assigned to the field in the straight respectively bent core, given a unit input in the straight waveguide at $z_{\mathrm{i}}$. Likewise $\left|S_{\mathrm{sb}}^{0}(z)\right|^{2}$ and $\left|S_{\mathrm{bb}}^{0}(z)\right|^{2}$ are the local powers in the straight and bent cores for a unit input in the bent waveguide at $\theta_{\mathrm{i}}$. Note that for the resonator description only the net effect of the entire coupler, i.e. the scattering matrix $S^{0}=S^{0}\left(z_{0}\right)$, is relevant.

\subsection{Projection onto the straight waveguide mode}

If the bend field radiates relatively heavily, it may take a long $z$-distance for the propagation and scattering matrix coefficients to stabilize; an oscillation may go on even beyond the rim of the ring. An example of such an oscillatory behaviour is given in Figure 3. These oscillations make the results seem untrustworthy; when the ring is sufficiently far away, the power in the straight waveguide should not vary. However, despite the oscillation, plots of the combined field seem to indicate that the modal power is actually rather constant already (see Figure 4), and that the fields are fairly close to those calculated by FDTD.

This leads to the idea of not just using the propagation matrix elements for the determination of the power transfer, but to consider the combined field and take the 

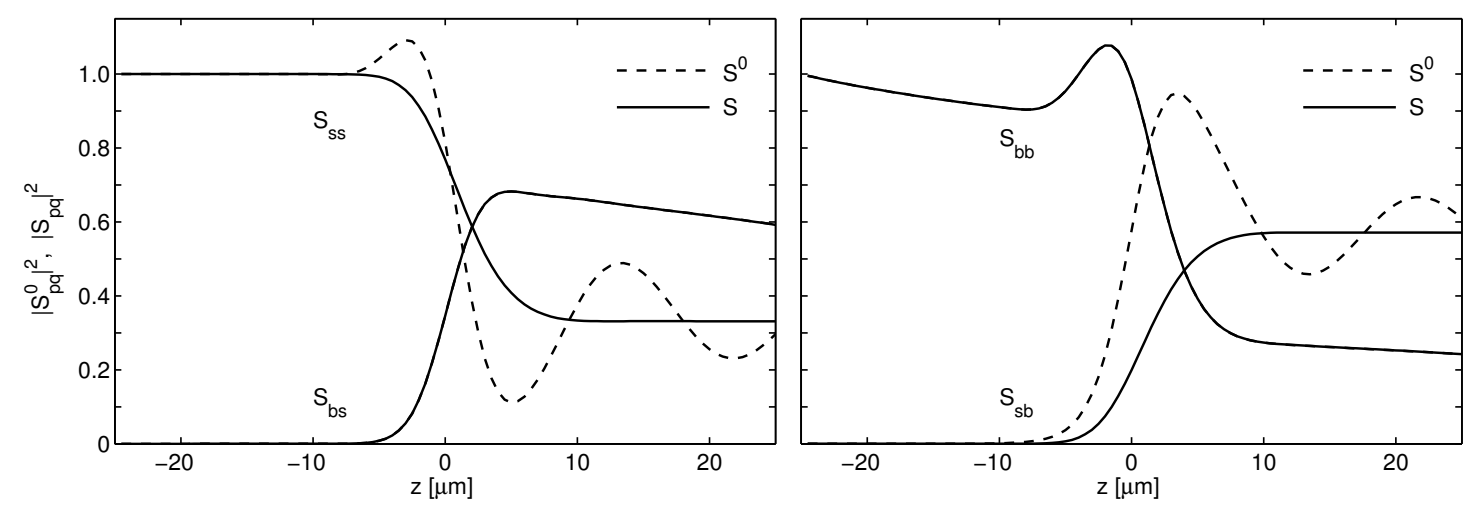

FIGURE 3. Evolution of the scattering matrix elements $S_{p q}^{0}$ and $S_{p q}$ (absolute squares) for a coupler structure as defined in Figure 1, for a bend radius $R=30 \mu \mathrm{m}$ and a narrow gap of width $g=0.1 \mu \mathrm{m}$. The CMT computational window is adjusted as described in Section 3, with $\Delta_{\mathrm{z}}=25, \Delta_{\mathrm{l}}=2 \mu \mathrm{m}$, and $\Delta_{\mathrm{r}}=20 \mu \mathrm{m}$. Note that the curves display coefficients for the interaction of non-orthogonal, not necessarily normalized basis fields.
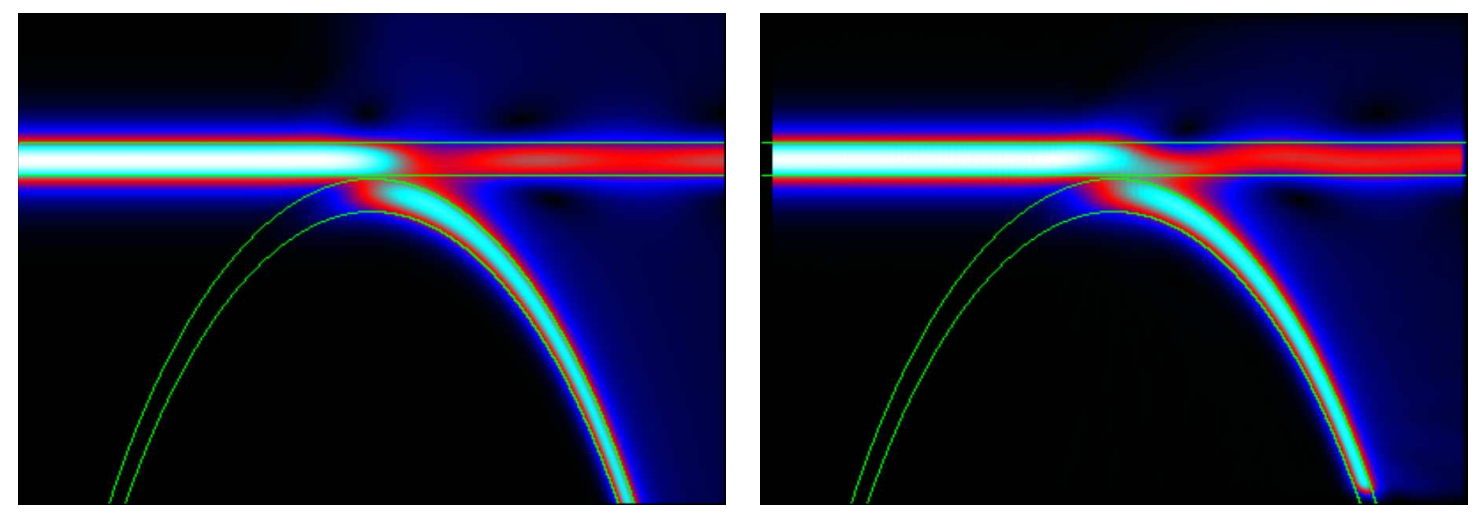

FIGURE 4. Absolute value field plots of CMT (left) and FDTD (right) calculations for the structure considered in Figure 3.

modal overlap with it, i.e. to project the total field onto the mode profile of the straight waveguide. In fact, these integrals are available already in the formulation (8): $\sigma_{\mathrm{ss}}$ is the overlap of the straight waveguide mode with itself, and $\sigma_{\mathrm{sb}}$ is the overlap of the straight waveguide mode with the bent waveguide mode. So, the corrected amplitude of the mode in the waveguide is extracted as

$$
A_{\mathrm{s}}(z)=\left(\frac{\sigma_{\mathrm{sb}}}{\sigma_{\mathrm{ss}}} a_{\mathrm{b}}(z)+a_{\mathrm{s}}(z)\right) \mathrm{e}^{-i \beta z} .
$$

It can be proven that the absolute value of this projected amplitude should become nearly constant. Consider the following derivative:

$$
\mathrm{d}_{z}\left(\sigma_{\mathrm{sb}} a_{\mathrm{b}}+\sigma_{\mathrm{ss}} a_{\mathrm{s}}\right)=\left(\sigma_{\mathrm{sb}} \mathrm{d}_{z} a_{\mathrm{b}}+\sigma_{\mathrm{ss}} \mathrm{d}_{z} a_{\mathrm{s}}+a_{\mathrm{b}} \mathrm{d}_{z} \sigma_{\mathrm{sb}}\right) .
$$

Note that the $z$-derivative of $\sigma_{\mathrm{ss}}$ is zero since the straight waveguide mode overlap with itself is constant. The first two terms on the right-hand side of Eq. (16) are equal to the 
bottom line of the left-hand side of the coupled mode equations (8), and can thus be replaced by the corresponding right-hand side. For the last term on the right-hand side, we can use the theorem (7) as follows:

$$
\begin{aligned}
\mathrm{d}_{z} \sigma_{\mathrm{sb}} & =\frac{1}{4} \int \mathbf{e}_{z} \cdot \mathrm{d}_{z}\left(\boldsymbol{E}_{\mathrm{b}} \times \boldsymbol{H}_{\mathrm{s}}^{*}+\boldsymbol{E}_{\mathrm{s}}^{*} \times \boldsymbol{H}_{\mathrm{b}}\right) \mathrm{d} x \\
& =\frac{1}{4} \int \nabla \cdot\left(\boldsymbol{E}_{\mathrm{b}} \times \boldsymbol{H}_{\mathrm{s}}^{*}+\boldsymbol{E}_{\mathrm{s}}^{*} \times \boldsymbol{H}_{\mathrm{b}}\right) \mathrm{d} x-\frac{1}{4} \int \mathbf{e}_{x} \cdot \mathrm{d}_{x}\left(\boldsymbol{E}_{\mathrm{b}} \times \boldsymbol{H}_{\mathrm{s}}^{*}+\boldsymbol{E}_{\mathrm{s}}^{*} \times \boldsymbol{H}_{\mathrm{b}}\right) \mathrm{d} x \\
& =-\mathrm{i} \frac{\omega \varepsilon_{0}}{4} \int \boldsymbol{E}_{\mathrm{s}}^{*} \cdot\left(\varepsilon_{\mathrm{b}}-\varepsilon_{\mathrm{s}}\right) \boldsymbol{E}_{\mathrm{b}} \mathrm{d} x-\frac{1}{4} \int \mathbf{e}_{x} \cdot \mathrm{d}_{x}\left(\boldsymbol{E}_{\mathrm{b}} \times \boldsymbol{H}_{\mathrm{s}}^{*}+\boldsymbol{E}_{\mathrm{s}}^{*} \times \boldsymbol{H}_{\mathrm{b}}\right) \mathrm{d} x
\end{aligned}
$$

Here $\mathbf{e}_{x}$ and $\boldsymbol{e}_{z}$ are unit vectors along the $x$ - and $z$-axes. Substituting Eq. (8) and Eq. (17) into Eq. (16), we get:

$$
\begin{aligned}
\mathrm{d}_{z}\left(\sigma_{\mathrm{sb}} a_{\mathrm{b}}+\sigma_{\mathrm{ss}} a_{\mathrm{s}}\right)= & -\mathrm{i} \frac{\omega \varepsilon_{0}}{4}\left(a_{\mathrm{b}} \int \boldsymbol{E}_{\mathrm{s}}^{*} \cdot\left(\varepsilon-\varepsilon_{\mathrm{s}}\right) \boldsymbol{E}_{\mathrm{b}} \mathrm{d} x+a_{\mathrm{s}} \int \boldsymbol{E}_{\mathrm{s}}^{*} \cdot\left(\varepsilon-\varepsilon_{\mathrm{s}}\right) \boldsymbol{E}_{\mathrm{s}} \mathrm{d} x\right) \\
& -a_{\mathrm{b}} \frac{1}{4} \int \mathbf{e}_{x} \cdot \mathrm{d}_{x}\left(\boldsymbol{E}_{\mathrm{b}} \times \boldsymbol{H}_{\mathrm{s}}^{*}+\boldsymbol{E}_{\mathrm{s}}^{*} \times \boldsymbol{H}_{\mathrm{b}}\right) \mathrm{d} x .
\end{aligned}
$$

Once the propagation is beyond the ring (ie, when $z>R$ and $\varepsilon=\varepsilon_{\mathrm{s}}$ ), or when the straight waveguide field is negligible in the bend, the only term left on the right-hand side is the last one, which reduces to a boundary term evaluated at the borders $x_{1}$ and $x_{\mathrm{r}}$ of the computational window. If the window is chosen large enough, the straight waveguide field will be negligible here as well, so the whole right-hand side of Eq. (18) becomes vanishingly small. Thus, the absolute value of $A_{\mathrm{s}}$ will tend to a constant value once the bend core is far enough separated from the straight waveguide.

Eq. (15) leads to a modified recipe for translating the solution $T$ of the coupled mode equations to the (definite, improved version of the) scattering matrix $\mathrm{S}$ :

$$
\mathrm{S}=\mathrm{O}_{\mathrm{o}} \mathrm{TO}_{\mathrm{i}}^{-1}
$$

with

$$
\mathrm{O}_{\mathrm{i}}=\left(\begin{array}{cc}
\mathrm{e}^{-\mathrm{i} \gamma_{\mathrm{b}} R \theta_{\mathrm{i}}} & 0 \\
\left.\left(\sigma_{\mathrm{sb}} / \sigma_{\mathrm{ss}}\right)\right|_{z_{\mathrm{i}}} & \mathrm{e}^{-\mathrm{i} \beta_{\mathrm{s}} z_{\mathrm{i}}}
\end{array}\right) \text { and } \mathrm{O}_{\mathrm{o}}=\left(\begin{array}{cc}
\mathrm{e}^{-\mathrm{i} \gamma_{\mathrm{b}} R \theta_{\mathrm{o}}} & 0 \\
\left.\left(\sigma_{\mathrm{sb}} / \sigma_{\mathrm{ss}}\right)\right|_{z_{\mathrm{o}}} & \mathrm{e}^{-\mathrm{i} \beta_{\mathrm{s}} z_{\mathrm{o}}}
\end{array}\right)
$$

Just as for $S^{0}$, one can consider the evolution of $S$ along $z$. Figure 3 includes corresponding curves for all four coefficients. As predicted, $S_{\mathrm{ss}}$ tends to a constant after the actual coupling region, as does $S_{\mathrm{sb}}$. This justifies the assumption of a limited interaction region even for the present example with pronounced radiation.

So far we did not incorporate a projection for extracting the external bend mode amplitude $A_{\mathrm{b}}$. For a sufficiently large computational window, the profile of the straight waveguide becomes negligible small in those regions of the radial port planes at $\theta_{\mathrm{i}}$ and $\theta_{\mathrm{o}}$ where the major part of the bend mode profile is located. Then the requirement of a continuous transition from the inner coupler region to the external field representation around the bend core leads to the zero entries in the upper right corners of $\mathrm{O}_{\mathrm{i}}$ and $\mathrm{O}_{\mathrm{o}}$. The projection does not alter the corresponding coefficents $S_{\mathrm{bb}}=S_{\mathrm{bb}}^{0}$ and $S_{\mathrm{bs}}=S_{\mathrm{bs}}^{0}$ that describe the power transfer to the bend mode. The scattering matrix includes the bend 
mode losses (i.e. relates the amplitudes $A_{\mathrm{b}}^{\mathrm{i}}$ and $A_{\mathrm{b}}^{\mathrm{o}}$ of identical bend mode profiles at $\theta=\theta_{\mathrm{i}}$ and $\theta=\theta_{\mathrm{o}}$ ), consequently the curves for the evolution of $S_{\mathrm{bb}}$ and $S_{\mathrm{bs}}$ in Figure 3 exhibit a regular decay outside the the interaction region.

\section{NUMERICAL RESULTS}

As a reference for the perturbational simulations we use a Finite Difference Time Domain program developed in our group that is based on the simple second-order Yee's mesh approach [3]. It is capable of TE or TM calculations on arbitrary structures in two spatial dimensions with (currently) real refractive indices and no dispersion. Perfectly matched layer (PML) boundary conditions border the rectangular computational window. As startfields, either CW or pulsed fields can be launched from the edge or from inside the computational window (using the so-called Total Field / Scattered Field approach). The results can be analyzed by means of modal overlaps, other power calculations, or field plots. The present FDTD calculations use a uniform discretization with a step size $0.05 \mu \mathrm{m}$ in the $x$ - and $z$-directions and a timestep of $1 \cdot 10^{-16} \mathrm{~s}$.

For the example system as defined in Figure 1, we perform calculations to assess the validity of the CMT theory. In Section 3.1 the direct transmission along the straight waveguide is compared between CMT and FDTD; Section 3.2 is concerned with the reciprocity properties of the CMT approximation. The CMT computational window $z_{\mathrm{i}}=-\Delta_{\mathrm{z}}, z_{\mathrm{o}}=\Delta_{\mathrm{z}}, x_{1}=\sqrt{\left(R-b-\Delta_{\mathrm{l}}\right)^{2}-\Delta_{\mathrm{z}}^{2}}, x_{\mathrm{r}}=R+\Delta_{\mathrm{r}}$ (cf. Figure 2) is specified by the length $\Delta_{z}$ of half the interval along $z$ and by radial and lateral borders $\Delta_{\mathrm{l}}$ and $\Delta_{\mathrm{r}}$ around the extremal positions of the bend core interfaces. Default values for the present structures are $\Delta_{\mathrm{l}}=2.0 \mu \mathrm{m}, \Delta_{\mathrm{r}}=20.0 \mu \mathrm{m}, \Delta_{\mathrm{z}}=R / 2$ if $R \leq 100 \mu \mathrm{m}$, otherwise $\Delta_{\mathrm{z}}=60 \mu \mathrm{m}$.

\subsection{Self coupling of the straight waveguide}

In order to compare the CMT to the FDTD results, a quantity must be taken that may be extracted from both methods. In the coupled mode theory, both the amplitudes in the straight core and the bend can be determined. In the FDTD method, the amplitude of the straight waveguide mode is determined by calculating the overlap integral of the local field with the mode profile. Defining the amplitude in the bent waveguide, however, is not quite as simple due to two reasons. Firstly, any overlap integrals are best calculated along either a horizontal or vertical cross-section, so the only place would be the horizontal line through the center of the ring. The fields are decoupled long before this position though, so a lot of calculation is done in areas where nothing of interest happens, unnecessarily increasing the computational effort. Secondly, since the bend mode radiates, the field may extend outward from the ring very far, which makes overlap integrals tricky to calculate unless the window is again made very large.

Another way to accurately estimate the straight-bend coupling coefficient is by considering a ring coupled to two parallel waveguides, one at the top and one at the bottom. If one then allows a pulse to travel from the top waveguide through the ring for about three quarters of a roundtrip, and measures the amplitude at the output of the lower waveguide, the pulse has passed through two couplers $\left(S_{b s}=S_{s b}\right.$, see Section 3.2). If one knows the decay due to the radiation of the bend mode, the coupling coefficient is 


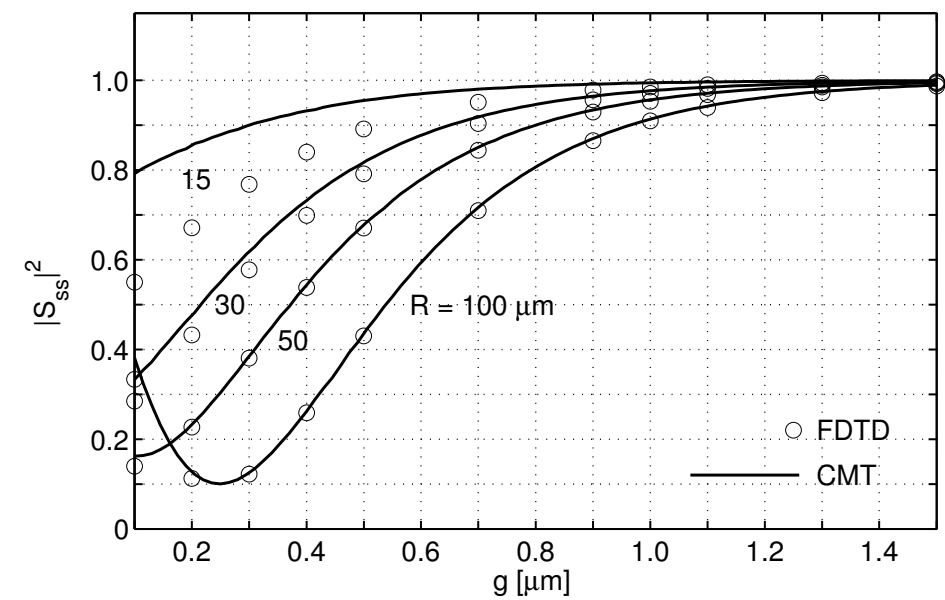

FIGURE 5. Amplitude $\left|A_{\mathrm{s}}^{o}\right|^{2}=$ $\left|S_{\mathrm{ss}}\right|^{2}$ of the straight waveguide mode in the output plane of the coupler structure for a unit excitation in the straight waveguide, calculated using both FDTD (circles) and CMT (lines). Parameters are as given in Figure 1, for bend radii of 15, 30, 50 and $100 \mu \mathrm{m}$ and a gap width $g$ varying between 0.1 and $1.5 \mu \mathrm{m}$.

easy to calculate. However, a large calculation window is again needed, and the wavelength dependence of the coupling coefficient would have to be taken into account.

Consequently the only element we will compare here is the self-coupling of the straight waveguide. The structure of Figure 1 was considered for various radii and gaps. Figure 5 shows the power in the straight guide at the coupler exit for both methods, given a normalized input in the straight core. In our CMT formulation, this quantity is represented by the coefficient $\left|S_{\mathrm{ss}}\right|^{2}$ of the coupler scattering matrix. One observes a very good agreement for radii down to about $30 \mu \mathrm{m}$, even for quite small gaps, where, due the violation of continuity conditions at the dielectric interfaces, the CMT approach becomes somewhat questionable.

\subsection{Reciprocity}

If only reciprocal (linear nonmagnetic) materials are involved, the coefficent for the transfer from one mode of one port waveguide of a system to the mode of another port waveguide has to be the same as the coefficent for the transfer from the second port to the first one $[8,5]$. First, we will give an example of reciprocity in FDTD; then, reciprocity will be checked for the coupled mode theory.

Figure 6 shows a straight waveguide coupled to a U-bend. This U-bend consists of a half-circle directly connected to straight waveguides. A mode is first launched into the top left-hand horizontal waveguide, and the power in the lower right-hand vertical waveguide is calculated; then, a mode is launched into the lower right-hand waveguide and the power in the top left-hand horizontal waveguide mode is calculated. Despite the pictures looking very different, reciprocity is satisfied; the power transfer is $12.9 \%$ in both cases.

If translated to our unidirectional coupler model, reciprocity (time reversal) and symmetry arguments as detailed in Ref. [5] require that the two complex offdiagonal (coupling) coefficients $S_{\mathrm{bs}}$ and $S_{\mathrm{sb}}$ are identical. For the symmetry argument to hold, identical mode profiles are to be used on the input and exit ports of the coupler, and the coupler interfaces need to be positioned symmetrically with respect to the $x$-axis, as realized by our choice of a symmetrical computational window. According to Figure 7, the approx- 

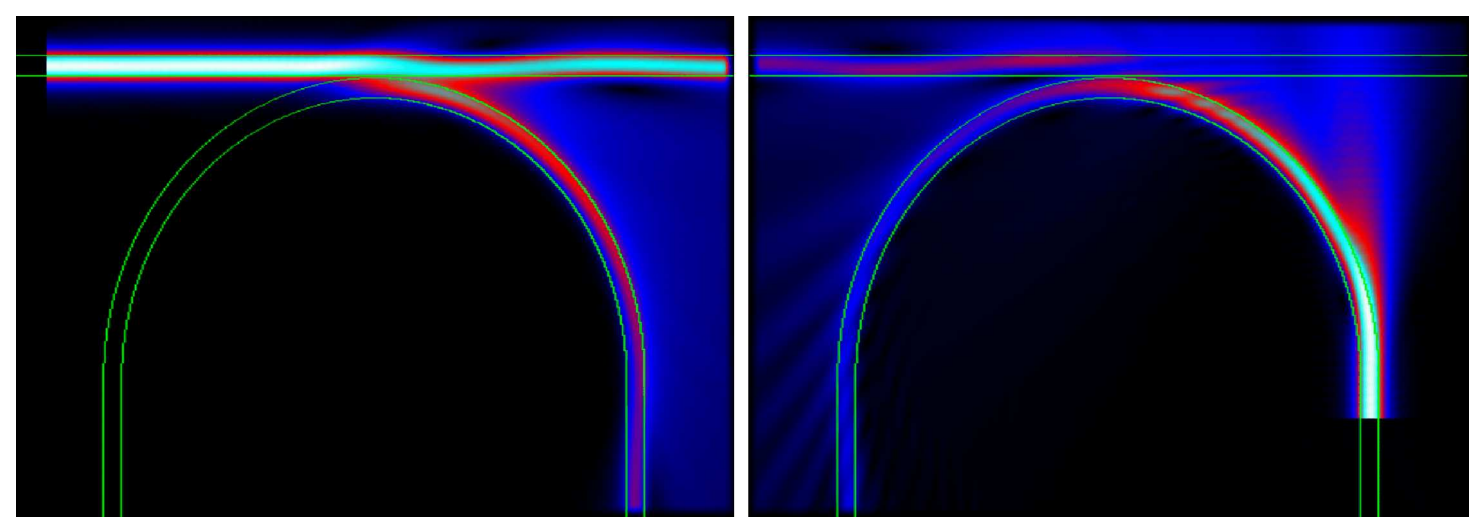

FIGURE 6. Absolute value field plots of FDTD calculations on a straight waveguide coupled to a Ubend with a radius of $R=15 \mu \mathrm{m}$ and a gap $g=0.1 \mu \mathrm{m}$. In the left-hand picture, the fundamental mode of the upper straight waveguide is launched; in the right-hand picture, the fundamental mode of the lower right-hand straight waveguide is launched vertically upwards.

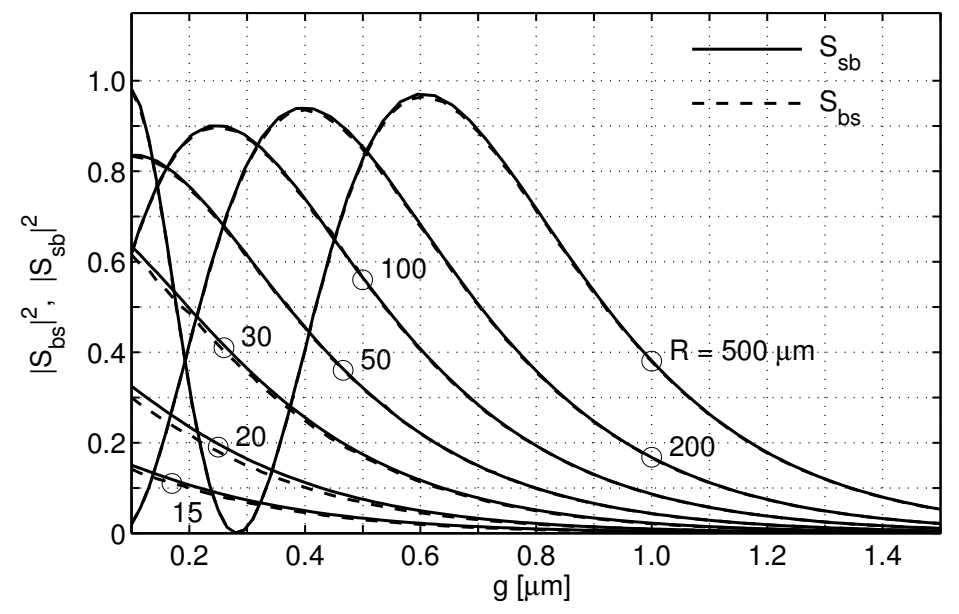

FIGURE 7. Absolute squares of the offdiagonal scattering matrix coefficients straight-to-bend $\left|S_{\mathrm{bs}}\right|^{2}$ and bend-to-straight $\left|S_{\mathrm{sb}}\right|^{2}$ as computed by coupled mode theory according to Eq. (19), for coupler structures as defined in Figure 1 with varying gap width $g$, for bend radii $R$ between 15 and $500 \mu \mathrm{m}$.

imate coupled mode formulation implements the reciprocity property remarkably well, at least what concerns the absolute value of the coupling coefficients. This applies even to regimes of rather narrow gaps and small bend radii with pronounced radiation, where one would have expected the inherent approximations to be invalid.

\section{CONCLUSIONS}

We have developed a coupled mode theory for the evanescent interaction between a straight waveguide and a circularly curved waveguide or disc. By transforming the bend mode profile, which is originally defined in cylindrical coordinates, to Cartesian coordinates the modes of the two systems can be linked by means of "standard" coupled mode theory. Since the mode of the bent waveguide is radiative, and thus has a complex propagation constant, the propagation matrix is not directly equal to the scattering matrix; apart from phase corrections the decay of the bend mode has to be taken into account when the scattering matrix elements are determined from a solution of the 
coupled mode equations.

Even when the bent and the straight cores are far apart, the overlap of the bend mode with the straight waveguide mode is not negligible for highly radiative bends. This causes the scattering matrix element related to the amplitude in the straight waveguide to oscillate heavily when viewed as a function of the position of the computational window borders. This applies also to extensions where one would have expected the interaction to be negligible. By taking the overlap of the composite field with the straight waveguide mode, this oscillation can be removed, yielding a constant amplitude of the straight waveguide mode after the actual coupling region. Apparently it is not adequate to view the CMT solution as an evolution of mode amplitudes along a propagation axis. One should consider the total CMT field as an approximation of a solution of Maxwell's equations on the domain given by the computational window and access only the modal amplitudes on the - more or less well defined - input and output ports.

Apart from the extent of the calculation window and the numerical evaluation of the integrals, the only real approximation in this formalism is the ansatz field (1). This field does not comply with Maxwell's equations in the regions where the index of refraction is different between the coupled structures due to a difference in phase velocity, and due to the fact that the interface conditions at material interfaces are not satisfied. This causes the CMT to fail for heavily radiating bend fields, which have a large amplitude in the straight waveguide region, while it is valid for lightly radiating fields; these comply with Maxwell's equations better since the the individual fields are much more confined to the area in which they are correct solutions. Even for small gaps, the fields of lightly radiating bends are small in the straight guide and vice-versa, which makes the CMT valid even for these cases.

Unfortunately, the variational principles behind the reciprocity techniques [8] do not provide a direct means to assess the quality of an approximate solution, even if a particular result is known to be optimal within the degrees of freedom incorporated in the field ansatz. The present ansatz field contains only two modes; the formalism might be improved, especially for highly radiative structures, by including higher-order modes of the bent or straight waveguides, or by including radiation in some way. However, comparison with FDTD fields shows that the two-mode ansatz describes the fields quite well already.

Comparisons of the transmission and coupling coefficients between FDTD and CMT calculations indicate that the CMT is indeed applicable for relatively heavily radiating structures, and for a rather strong interaction in case of narrow core distances. In conclusion, the present formulation of coupled mode theory leads to a simple, fast, and (unexpectedly) trustworthy tool for the simulation and design of coupler configurations for ringresonator devices.

\section{ACKNOWLEDGMENTS}

This work is supported by the European Commission (project IST-2000-28018, 'NAIS' [13]). The authors thank E. van Groesen and H. J. W. M. Hoekstra for many fruitful discussions. 


\section{REFERENCES}

1. Driessen, A., Geuzebroek, D., Hoekstra, H. J. W. M., Kelderman, H., Klein, E. J., Klunder, D. J. W., Roeloffzen, C. G. H., Tan, F. S., Krioukov, E., Otto, C., Gersen, H., van Hulst, N. F., and Kuipers, L., Microresonators as building blocks for VLSI photonics (2004), this volume.

2. Little, B. E., Chu, S. T., Haus, H. A., Foresi, J., and Laine, J.-P., Journal of Lightwave Technology, 15, 998-1005 (1997).

3. Taflove, A., and Hagness, S. C., Computational Electrodynamis: The Finite Difference Time Domain Method, 2nd ed., Artech House, Norwood, MA, USA, 2000.

4. Okamoto, K., Fundamentals of Optical Waveguides, Academic Press, SanDiego, 2000.

5. Hammer, M., Hiremath, K. R., and Stoffer, R., Analytical approaches to the description of optical microresonator devices (2004), this volume.

6. Chuang, S. L., Journal of Lightwave Technology, 5, 5-15 (1987).

7. Haus, H. A., Huang, W. P., Kawakami, S., and Whitaker, N. A., Journal of Lightwave Technology, LT-5, 16-23 (1987).

8. Vassallo, C., Optical Waveguide Concepts, Elsevier, Amsterdam, 1991.

9. Rowland, D. R., and Love, J. D., IEE Proceedings, Pt. J, 140, 177-188 (1993).

10. Klunder, D. J. W., Tan, F. S., van der Veen, T., Bulthuis, H. F., Sengo, G., Docter, B., Hoekstra, H. J. W. M., and Driessen, A., Journal of Lightwave Technology, 21, 1099-1110 (2003).

11. Hiremath, K. R., Modeling of 2D cylindrical optical microresonators (2003), MSc Thesis, University of Twente, Enschede, The Netherlands.

12. Press, W. H., Teukolsky, S. A., Vetterling, W. T., and Flannery, B. P., Numerical Recipes in C, 2nd ed., Cambridge University Press, 1992.

13. NAIS, Next-generation active integrated optic subsystems (project start: 2001), Information society technologies programme of the European Commission, project IST-2000-28018, http://www.mesaplus.utwente.nl/nais/. 